\title{
Comparing photon and proton-based hypofractioned SBRT for prostate cancer accounting for robustness and realistic treatment deliverability
}

\author{
${ }^{1}$ LEE C GODDARD, MPhys, ${ }^{1,2} \mathrm{~N}$ PATRIK BRODIN, PhD, ${ }^{1}$ WILLIAM R BODNER, MD, ${ }^{1}$ MADHUR K GARG, MD and \\ ${ }^{1,2}$ WOLFGANG A TOMÉ, PhD, FAAPM, FASTRO \\ ${ }^{1}$ Department of Radiation Oncology, Montefiore Medical Center, Bronx, NY, United States \\ ${ }^{2}$ Institute for Onco-Physics, Department of Radiation Oncology, Albert Einstein College of Medicine, Bronx, NY, United States \\ Address correspondence to: Prof Wolfgang A Tomé \\ E-mail: wolfgang.tome@einstein.yu.edu
}

Objective: To investigate whether photon or proton-based stereotactic body radiation therapy (SBRT is the preferred modality for high dose hypofractionation prostate cancer treatment. Achievable dose distributions were compared when uncertainties in target positioning and range uncertainties were appropriately accounted for.

Methods: 10 patients with prostate cancer previously treated at our institution (Montefiore Medical Center) with photon SBRT using volumetric modulated arc therapy (VMAT) were identified. MRI images fused to the treatment planning $C T$ allowed for accurate target and organ at risk (OAR) delineation. The clinical target volume was defined as the prostate gland plus the proximal seminal vesicles. Critical OARs include the bladder wall, bowel, femoral heads, neurovascular bundle, penile bulb, rectal wall, urethra and urogenital diaphragm. Photon plan robustness was evaluated by simulating $2 \mathrm{~mm}$ isotropic setup variations. Comparative proton SBRT plans employing intensity modulated proton therapy (IMPT) were generated using robust optimization. Plan robustness was evaluated by simulating $2 \mathrm{~mm}$ setup variations and $3 \%$ or $1 \%$ Hounsfield unit (HU) calibration uncertainties.

Results: Comparable maximum OAR doses are achievable between photon and proton SBRT, however, robust optimization results in higher maximum doses for proton SBRT. Rectal maximum doses are significantly higher for Robust proton SBRT with $1 \%$ HU uncertainty compared to photon SBRT ( $p=0.03$ ), whereas maximum doses were comparable for bladder wall $(p=0.43)$, urethra $(p=0.82)$ and urogenital diaphragm $(p=0.50)$. Mean doses to bladder and rectal wall are lower for proton SBRT, but higher for neurovascular bundle, urethra and urogenital diaphragm due to increased lateral scatter. Similar target conformality is achieved, albeit with slightly larger treated volume ratios for proton SBRT, >1.4 compared to 1.2 for photon SBRT.

Conclusion: Similar treatment plans can be generated with IMPT compared to VMAT in terms of target coverage, target conformality, and OAR sparing when range and $\mathrm{HU}$ uncertainties are neglected. However, when accounting for these uncertainties during robust optimization, VMAT outperforms IMPT in terms of achievable target conformity and OAR sparing.

Advances in knowledge: Comparison between achievable dose distributions using modern, robust optimization of IMPT for high dose per fraction SBRT regimens for the prostate has not been previously investigated.

\section{INTRODUCTION}

Prostate cancer accounts for almost $11 \%$ of new cancer diagnoses in the United States each year and is the sixth leading cause of cancer deaths. ${ }^{1}$ As such, it is the subject of extensive research efforts and ever changing trends in treatment. Some of these changes have been motivated by radiobiological evidence showing that the $\alpha / \beta$ ratio of prostate cancer can be as low as $1.5 \mathrm{~Gy},{ }^{2-4}$ supporting the use of a hypofractionated dose regimen. The goal of hypofractionation in prostate cancer treatment is improved local control, whilst maintaining similar rates of normal tissue toxicities. Stereotactic body radiation therapy (SBRT) allows for the precise deposition of dose required for these hypofractionated treatments and is becoming increasingly common. ${ }^{5,6}$ Increasing the dose delivered to the target may improve patient outcomes; however, this comes with increased risk of normal tissue toxicity. A number of Phase I dose escalation trials have 
determined that dose regimens up to $50 \mathrm{~Gy}$ in five fractions are safe and deliverable using photon SBRT. ${ }^{7,8}$

Proton therapy is also becoming more common in the treatment of prostate cancer, as it too allows for precise dose deposition within the target volume with the advantage of a lower integral dose to surrounding normal tissues. ${ }^{5}$ It has been shown that passive scattering proton techniques are feasible when using a hypofractionated dose, ${ }^{9}$ however, the additional uncertainties inherent to the delivery of proton therapy were not taken into account.

SBRT and proton therapy share common uncertainties in treatment delivery, these include target definition, target motion, normal tissue motion and patient setup. In addition, proton therapy must consider range uncertainties due to the conversion of electron densities, measured using a CT scan, to proton stopping powers. These uncertainties lead to the delivered dose distribution varying from the intended distribution and have the potential to result in considerable variations in the delivered dose distribution. ${ }^{10}$

This study directly compares the achievable dose distributions of photon SBRT delivered using volumetric modulated arc therapy (VMAT), to proton SBRT delivered using intensity modulated proton therapy (IMPT), when uncertainties in target positioning and range uncertainties are appropriately accounted for. This, in order to investigate which modality would be the preferred option for hypofractionated prostate cancer radiation therapy.

\section{METHODS AND MATERIALS}

\section{Patient material and SBRT protocol}

10 patients with prostate cancer previously treated at our institution in 2016/17 were identified, 1 low-risk, 6 intermediate-risk and 3 high-risk disease patients. CT simulation was obtained using a $1.25 \mathrm{~mm}$ slice thickness across the target region; oral contrast (Gastrografin, Bracco Diagnostic Imaging, Monroe Township, NJ) was administered 20 min prior to simulation. A $3 \mathrm{~T} T_{1}$ weighted threedimensional VISTA (Phillips, Amsterdam, Netherlands) MRI was acquired immediately following the CT to minimize anatomical differences such a bowel and bladder filling between images. MRI images were co-registered to the treatment planning CT using a rigid registration of soft tissues, to allow for accurate target and organ at risk (OAR) delineation. OARs were delineated based on the MRI images and then adjusted as necessary based on the treatment planning CT. For each patient, the clinical target volume (CTV) was defined as the prostate gland plus the proximal seminal vesicles. CTV volume ranged from 32.1 to $74.3 \mathrm{~cm}^{3}$ with a mean volume of $46.9 \mathrm{~cm}^{3}$. A $2 \mathrm{~mm}$ uniform expansion was used to generate a planning target volume (PTV). A dose prescription volume (DPV) was created by removing all overlapping OARs, plus a $2 \mathrm{~mm}$ margin, from the PTV. Critical OARs contoured for this treatment protocol include the bladder wall, bowel, femoral heads, neurovascular bundle, penile bulb, rectal wall, urethra, and urogenital diaphragm.

An endorectal balloon, inflated with $100-150 \mathrm{~cm}^{3}$ of air, was placed for imaging and treatment to fixate the prostate against
Figure 1. Example dose distributions for a VMAT (a) and $1 \%$ HU Robust IMPT (b) treatment plan. HU, Hounsfield unit; IMPT, intensity modulated proton therapy; VMAT, volumetric modulated arc therapy.

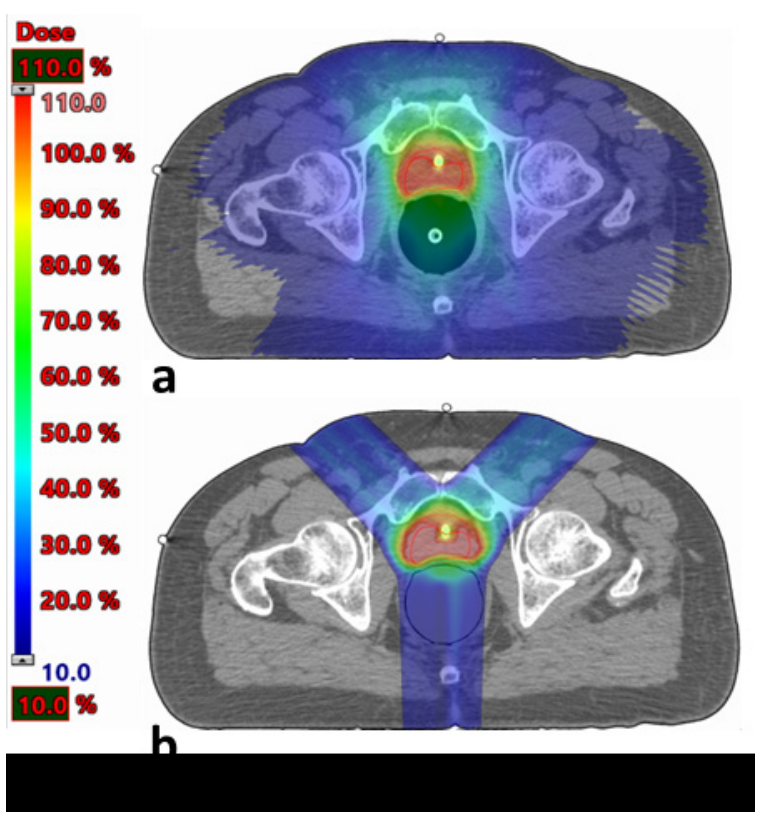

the pubic symphysis, minimizing target motion and reducing dose to the rectal wall. ${ }^{11}$ A Foley catheter placed at imaging and treatment allows for consistent bladder fill. Calypso fiducials (Varian Medical Systems, Palo Alto, CA) were also used to allow for continuous intrafraction target tracking. Daily image guidance, in the form of cone beam CT scans and orthogonal $\mathrm{kV}$ images, was used to minimize interfraction setup variations.

\section{Photon treatment planning}

Patients were treated using a Trilogy LINAC (Varian Medical Systems, Palo Alto, CA) equipped with a high definition multileaf collimator (MLC). Treatment plans were generated using Eclipse v.11.0 treatment planning system (Varian Medical Systems, Palo Alto, CA) using the AAA v.11.0.31 calculation algorithm. Treatment plans were generated using three $360^{\circ}$ VMAT fields cf. Figure 1a. The field size was set to cover the PTV plus a $2 \mathrm{~mm}$ margin. Beam energy was set to "6X-SRS" mode, allowing for a dose rate of 1000 monitor units per minute to be used, shortening treatment delivery time and minimizing intrafraction motion. VMAT plans were normalized, so that the prescription dose covered $95 \%$ of the DPV structure. Plan robustness was evaluated by simulating $2 \mathrm{~mm}$ setup variations in all directions and recalculating the dose distribution for each isocenter shift. Hence, for each patient six "uncertainty" dose distributions were also generated in addition to the "nominal" dose distribution.

\section{IMPT treatment planning}

IMPT plans using several beam arrangements were considered for each patient. Treatment plans were generated using Eclipse v.13.7 treatment planning system (Varian Medical Systems, Palo Alto, CA) using the CAPPCS v.13.7.15 calculation algorithm and the CAPNUPO v.13.7.15 multifield optimization algorithm. The optimal beam arrangement was determined to be a 3-field 
Y-shaped arrangement with a posteroanterior, right anterior oblique and left anterior oblique field, cf. Figure $1 \mathrm{~b}$. This beam arrangement was used for each patient. Field specific PTVs were generated using a $2 \mathrm{~mm}$ margin from the CTV for target motion and a 3\% Hounsfield unit (HU) calibration uncertainty, typical of current clinical practice. ${ }^{12}$ Plans were also generated using a $1 \% \mathrm{HU}$ uncertainty to represent potential improvements of proton stopping power calculations. This reduced uncertainty has been shown to be achievable using dual energy CT and proton $\mathrm{CT},{ }^{13,14}$ however, the clinical use of these imaging modalities is not currently widespread. IMPT plans were also generated using the robust planning feature in Eclipse, which generates the optimal fluence taking target positioning and $\mathrm{HU}$ calibration uncertainties into account. Robust objectives were used for all OAR maximum doses and $1 \mathrm{cc}$ dose objectives as well as D95\% objectives for the CTV. The density of the air-filled rectal balloon used in photon planning was overridden and set to water to minimize dosimetric uncertainties. ${ }^{15}$ IMPT plans were normalized, so that the prescription dose covered $95 \%$ of the CTV, minus a $2 \mathrm{~mm}$ margin from any overlapping OARs, referred to as the $\mathrm{CTV}_{\text {Eval }}$. Plan robustness was evaluated by simulating $2 \mathrm{~mm}$ setup variations and 3 or $1 \% \mathrm{HU}$ calibration uncertainties. Hence, for each patient, 12 "uncertainty" dose distributions were generated in addition to the nominal dose distribution. The range of doses to each OAR and the target volume coverage was calculated for photon and IMPT treatment plans respectively.

\section{Plan evaluation}

Target dose coverage was analyzed by recording the volume of the $\mathrm{CTV}_{\text {Eval }}$ receiving prescription dose $\left(\mathrm{V}_{100 \%}\right)$. The maximum dose $\left(D_{\max }\right)$ and mean dose $\left(D_{\text {mean }}\right)$ delivered to each OAR was recorded for each planning technique for nominal and all uncertainty dose distributions. Several parameters defined below were also used to further compare plan quality of the nominal dose distributions only.

$$
\begin{aligned}
& T V R=\frac{V_{100 \%}}{V_{C T V}} \\
& C V R=\frac{V_{50 \%}}{V_{C T V}}
\end{aligned}
$$

The treated volume ratio (TVR) and clinically significant volume ratio (CVR) are defined as the volume of tissue receiving $100 \%$ of the prescription dose $\left(\mathrm{V}_{100 \%}\right)$ and the volume receiving $50 \%$ of the prescription dose $\left(\mathrm{V}_{50 \%}\right)$ to the CTV volume $\left(\mathrm{CTV}_{\mathrm{Vol}}\right)$, respectively.

$$
H I=\frac{D_{2 \%}-D_{98 \%}}{D_{R x}}
$$

The homogeneity index (HI), defined as the maximum dose to $\geq 2 \%$ of the target $\left(D_{2 \%}\right)$ minus the minimum dose to $\leq 98 \%$ of the target $\left(D_{98 \%}\right)$, divided by the prescription dose $\left(D_{R x}\right)$ was also calculated for nominal dose distributions. ${ }^{16}$

$$
\begin{gathered}
C G I_{c}=\frac{V_{D P V}}{V_{100 \%}} \\
C G I_{b}=1-\left[\frac{r_{50 \%}-r_{100 \%}-\Delta r_{b}}{\Delta r_{b}}\right]
\end{gathered}
$$

$$
C G I=0.5\left[C G I_{c}+C G I_{b}\right]
$$

The conformity gradient index (CGI) is defined as the average of $\mathrm{CGI}_{\mathrm{c}}$ and the gradient benchmark index $\left(\mathrm{CGI}_{\mathrm{b}}\right){ }^{17} \mathrm{CGI}_{\mathrm{c}}$ is defined as the ratio of the volume of the treatment modality specific DPV ( $\mathrm{V}_{\mathrm{DPV}}$ ) to $\mathrm{V}_{100 \%}$ such that a value of one represents a target perfectly covered by the $D_{R x}$, values less than one indicate an overcovered target and larger than one indicate an undercovered target. $r_{50 \%}$ and $r_{100 \%}$ are the effective radii of the 50 and $100 \%$ isodose volumes respectively. The expected, or benchmark, fall-off distance $\left(\Delta r_{b}\right)$ from 100 to $50 \%$ is dependent on the treatment modality and disease site. A value of $20 \mathrm{~mm}$ was used in this study as commonly found in SBRT. CGI $\mathrm{b}_{\mathrm{b}}$ allows for comparison of the achieved dose gradient to the benchmark gradient. A value of one represents a dose fall-off equal to benchmark, values less than 1 indicate worse than benchmark and values larger than 1 indicate better than benchmark.

\section{Statistical analysis}

The $95 \%$ confidence interval in $D_{\max }$ and $D_{\text {mean }}$ for each patient was calculated based on the variation introduced by the uncertainty simulations. Statistically significant differences in the nominal OAR $\mathrm{D}_{\max }$ and $\mathrm{D}_{\text {mean }}$ doses between the VMAT and IMPT plans were assessed using paired Wilcoxon signed-rank tests, with $p<0.05$ considered statistically significant.

The 95\% confidence interval for the TVR, CVR, HI and CGI indices were calculated based on the variation in the nominal plans amongst patients.

\section{RESULTS}

For all patients, the dose to the bowel, femoral heads and penile bulb were well below the constraints outlined in the protocol for both VMAT and IMPT plans, and as such these were excluded from further analysis. Of note, doses to these OARs were typically lower for IMPT plans due to the lower integral dose associated with this modality.

Table 1 shows the average $D_{\max }$ for each OAR for the patients included in the analysis. Whilst comparable doses are achievable with IMPT for both the 3 and 1\% HU uncertainty plans, robust optimization results in a higher average $\mathrm{D}_{\max }$ compared to the VMAT plans. As expected, the robust optimization reduces the plan uncertainty dose range, as reflected in the smaller $95 \%$ confidence interval for the robust IMPT plans. This is more apparent for the $3 \%$ as compared to the $1 \%$ HU uncertainty.

Table 2 shows the average $D_{\text {mean }}$. Dose to both the bladder and rectal wall is lower for all IMPT plans with a lower mean dose in the $1 \% \mathrm{HU}$ uncertainty plans. The mean dose to the neurovascular bundle, urethra and urogenital diaphragm is higher for all IMPT plans due to the larger lateral scatter of this technique.

Table 3 shows the average TVR, CVR, HI, CGI ${ }_{c}, C_{b}$ and CGI. VMAT and IMPT have similar $\mathrm{CGI}_{c}$ values; however, $\mathrm{CGI}_{c}$ decreases for robust plans meaning the conformity is worse. By calculating the TVR, it can be seen that for IMPT plans, the conformity to the CTV is worse. It should be noted that due to 
Table 1. Average $D_{\max }$ as a percentage of prescription dose for the various OARs with $\pm 95 \%$ confidence interval

\begin{tabular}{|l|c|c|c|c|c|}
\hline Organ at risk & VMAT & IMPT 3\% HU & IMPT 3\% HU robust & IMPT 1\% HU & IMPT 1\% HU robust \\
\hline Bladder wall & $98.7 \pm 4.6$ & $98.6 \pm 3.0$ & $106.2 \pm 3.6$ & $98.9 \pm 2.1$ & $100.4 \pm 2.3$ \\
\hline Neurovascular bundle & $101.3 \pm 3.0$ & $100.7 \pm 3.0$ & $107.6 \pm 1.4$ & $101.9 \pm 2.3$ & $103.0 \pm 1.9$ \\
\hline Rectal wall & $88.7 \pm 5.5$ & $91.4 \pm 12.7$ & $94.0 \pm 4.0$ & $91.7 \pm 4.6$ & $93.4 \pm 4.1$ \\
\hline Urethra & $92.0 \pm 3.2$ & $92.7 \pm 13.7$ & $97.2 \pm 3.3$ & $92.6 \pm 5.2$ & $92.7 \pm 2.2$ \\
\hline Urogenital diaphragm & $79.8 \pm 13.0$ & $93.5 \pm 5.7$ & $94.6 \pm 4.6$ & $93.1 \pm 4.7$ & $89.8 \pm 4.8$ \\
\hline
\end{tabular}

HU, Hounsfield unit; IMPT, intensity modulated proton therapy; OAR, organ at risk; VMAT, volumetric modulated arc therapy.

the added HU uncertainty, the field-specific PTVs for IMPT plans are larger than for VMAT plans, with the $3 \%$ HU uncertainty resulting in the largest PTV and hence, the largest TVR. The CVR is superior for IMPT plans and is a result of the reduced integral dose achievable with IMPT. The HI values for nonrobust IMPT plans are slightly worse than for VMAT, indicating large inhomogeneity within the PTV. The HI is greatly increased for robust plans, where homogeneity is sacrificed to allow for more robust dose distributions. $\mathrm{CGI}_{\mathrm{b}}$ values for all plans were greater than 1, indicating dose falloff was better than the baseline value of $20 \mathrm{~mm}$, with improved falloff seen in the IMPT plans.

Figure 2 shows the $\mathrm{D}_{\max }$ for each patient included in the analysis comparing VMAT plans to IMPT plans using a $1 \% \mathrm{HU}$ calibration uncertainty. Data points above the dashed line indicate higher OAR doses from IMPT compared to VMAT, and vice versa. It can be seen that rectal $D_{\max }$ doses are significantly higher for IMPT $(p=0.03)$, and somewhat higher for neurovascular bundle, although not statistically significant $(p=0.11)$, whereas $\mathrm{D}_{\max }$ doses were comparable for bladder wall $(p=0.43)$, urethra $(p=0.82)$ and urogenital diaphragm $(p=0.50)$.

Table 4 shows the $\mathrm{CTV}_{\text {Eval }} \mathrm{V}_{100 \%}$ values between the various treatment plans. IMPT plans were normalized, so the prescription dose covers $95 \%$ of the $\mathrm{CTV}_{\text {Eval }}$ volume. Slightly higher target coverage was achievable with VMAT plans, likely due to the fact that these were optimized to a PTV structure encompassing the $\mathrm{CTV}_{\text {Eval }}$. Importantly, robust optimization greatly reduces the variance in target coverage for the IMPT plans, illustrated by the smaller $95 \%$ confidence intervals.

\section{DISCUSSION}

Despite advances in proton therapy delivery techniques and imaging that have allowed for more precise treatment planning and delivery, photon-based SBRT allows for improved sparing of normal tissues adjacent to the target in the critical high-dose region. It also allows a smaller volume of tissue to be treated to the prescription dose, as there is no need for stopping power conversions and the added uncertainties they bring. Robust optimization options in proton treatment planning reduce this uncertainty; however, we have shown that the OAR $D_{\max }$ is increased when robust planning is utilized. As SBRT technologies have matured, higher doses per fraction have been shown to be safe. ${ }^{7,8}$ At these high doses per fraction, a larger margin from critical structures is required for IMPT compared to VMAT to achieve acceptable doses to normal tissues, resulting in a lower dose to areas of the prostate CTV. The need for larger margins between target volume and OAR in the case of IMPT could be achieved through the use of tissue expanders/separators between them allowing the safe use of IMPT.

A previous study by Kole et al compared passively scattered proton therapy to photon SBRT using the Cyberknife platform for 10 patients treated to a dose of 36.25 Gy in five fractions. ${ }^{9}$ Using a $2.5 \%+1.5 \mathrm{~mm}$ HU calibration uncertainty, they too found improved target conformity with photon SBRT, but found a reduced dose to the penile bulb when using proton therapy. They also found that the mean dose to the femoral heads was higher when using proton therapy, due to the right lateral and left lateral beam arrangement they employed. Comparing $\mathrm{D}_{\max }$ of the various OARs, they found little difference between the two modalities, analogous to the findings in our study.

Our study assumes the same patient setup techniques would be used for the two modalities, with the exception of the rectal balloon being filled with water instead of air for proton planning (simulated in the current study by overriding this structure as water-equivalent for the proton plans). The advantages of using the air-filled rectal balloon for photon planning are twofold. Firstly, the rectal balloon fixates the

Table 2. Average $D_{\text {mean }}$ as a percentage of prescription dose for the various OARs with $\pm 95 \%$ confidence interval

\begin{tabular}{|l|c|c|c|c|c|}
\hline Organ at risk & VMAT & IMPT 3\% HU & IMPT 3\% HU robust & IMPT 1\% HU & IMPT 1\% HU robust \\
\hline Bladder wall & $32.3 \pm 1.0$ & $28.2 \pm 0.6$ & $29.1 \pm 0.6$ & $26.3 \pm 0.5$ & $26.5 \pm 0.5$ \\
\hline Neurovascular bundle & $82.6 \pm 1.9$ & $97.4 \pm 1.9$ & $101.1 \pm 1.5$ & $90.7 \pm 1.3$ & $92.3 \pm 0.9$ \\
\hline Rectal wall & $33.1 \pm 0.6$ & $28.9 \pm 0.1$ & $32.4 \pm 0.4$ & $27.04 \pm 0.4$ & $28.4 \pm 0.3$ \\
\hline Urethra & $47.5 \pm 0.7$ & $58.5 \pm 3.9$ & $61.2 \pm 0.6$ & $58.6 \pm 1.7$ & $59.0 \pm 0.5$ \\
\hline Urogenital diaphragm & $27.5 \pm 2.5$ & $44.6 \pm 2.0$ & $44.6 \pm 1.8$ & $42.4 \pm 1.9$ & $41.9 \pm 1.6$ \\
\hline
\end{tabular}

HU, Hounsfield unit; IMPT, intensity modulated proton therapy; OAR, organ at risk; VMAT, volumetric modulated arc therapy. 
Table 3. Conformity metrics comparing VMAT to IMPT plans with $\pm 95 \%$ confidence interval for the TVR, CVR, HI and conformity gradient indices

\begin{tabular}{|l|c|c|c|c|c|}
\hline & VMAT & IMPT 3\% HU & IMPT 3\% HU robust & IMPT 1\% HU & IMPT 1\% robust \\
\hline TVR & $1.22 \pm 0.09$ & $1.62 \pm 0.22$ & $1.75 \pm 0.21$ & $1.41 \pm 0.15$ & $1.42 \pm 0.12$ \\
\hline CVR & $6.49 \pm 0.91$ & $5.00 \pm 0.67$ & $5.02 \pm 0.66$ & $4.30 \pm 0.53$ & $4.23 \pm 0.46$ \\
\hline $\mathrm{HI}$ & $0.09 \pm 0.01$ & $0.15 \pm 0.01$ & $0.39 \pm 0.04$ & $0.15 \pm 0.01$ & $0.29 \pm 0.02$ \\
\hline $\mathrm{CGI}_{\mathrm{c}}$ & $0.96 \pm 0.02$ & $0.95 \pm 0.03$ & $0.88 \pm 0.05$ & $0.95 \pm 0.03$ & $0.94 \pm 0.04$ \\
\hline $\mathrm{CGI}_{\mathrm{b}}$ & $1.13 \pm 0.07$ & $1.41 \pm 0.02$ & $1.45 \pm 0.02$ & $1.45 \pm 0.02$ & $1.46 \pm 0.03$ \\
\hline $\mathrm{CGI}$ & $1.05 \pm 0.04$ & $1.18 \pm 0.02$ & $1.16 \pm 0.03$ & $1.20 \pm 0.02$ & $1.20 \pm 0.03$ \\
\hline
\end{tabular}

CGI, conformity gradient index; CVR, clinically significant volume ratio; HI, homogeneity index; TVR, treated volume ratio.

prostate against the pubic symphysis, reducing intrafraction motion of the prostate and allowing for the small PTV expansion. Secondly, the expansion of the balloon causes the rectal wall to thin, conforming to the air cavity of the balloon. Thus, dose to the rectal wall is slightly reduced due to a build-down region attributable to the air cavity inside the balloon. For IMPT planning, the presence of the air cavity causes large dosimetric uncertainties, so simulated water filling was used as this is dosimetrically reproducible, whilst still allowing for target fixation. ${ }^{18}$ The beam arrangement chosen for IMPT planning was found to offer the optimum target coverage and OAR sparing, however, this may not represent the best clinical practice depending on institutional variability.

Rectal spacer gels are increasingly being used to increase the distance between the prostate and rectum, allowing for greater sparing of the rectal wall. Although this may result in favorable rectal doses, when compared to the use of an endorectal balloon, intrafraction prostate motion would be increased, resulting in the need for larger PTV expansion and increased dose to other normal tissues. The use of a spacer hydrogel that hardens and stays in place after administration, such as SpaceOAR (Augmenix, Bedford, MA), in conjunction with an endorectal balloon may allow for greater sparing and rigid fixation of the

Figure 2. Average OAR $D_{\max }$ values for VMAT and IMPT plans, with error bars representing $95 \%$ confidence intervals calculated based on variability between patients and variability attributable to robustness. IMPT, intensity modulated proton therapy; OAR, organ at risk; VMAT, volumetric modulated arc therapy.
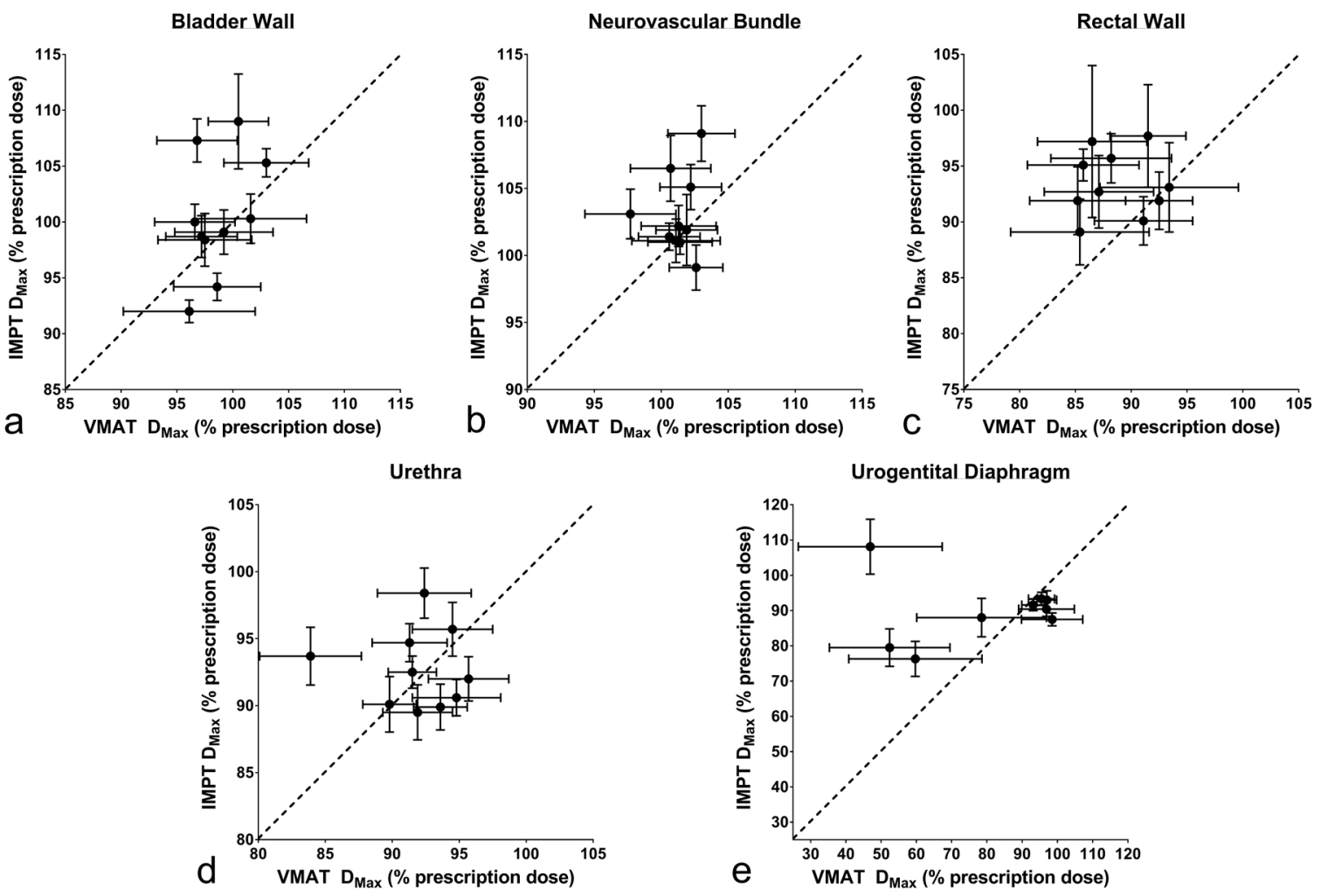
Table 4. Average CTV $\vee_{\text {Eval }} \vee_{100 \%}$ for VMAT and IMPT plans with $\pm 95 \%$ confidence intervals

\begin{tabular}{|c|c|c|c|c|}
\hline VMAT & IMPT 3\% HU & IMPT 3\% HU robust & IMPT 1\% HU & IMPT 1\% robust \\
\hline $97 \pm 2.36$ & $95 \pm 11.66$ & $95 \pm 2.00$ & $95 \pm 4.63$ & $95 \pm 1.89$ \\
\hline
\end{tabular}

CTV, clinical target volume; HU, Hounsfield unit; IMPT, intensity modulated proton therapy; VMAT, volumetric modulated arc therapy.

prostate during treatment allowing one to substantially improve rectal wall dosimetry.

Recent studies have shown that the relative biological effectiveness (RBE) of proton therapy, typically assumed to be 1.1, may in fact vary based upon the fractionation schedule and tissue type. ${ }^{19}$ For the bladder and rectal wall, a RBE of 1.2 for SBRT treatments may be more appropriate. Normal Tissue Complication Probabilty (NTCP) estimates based on using a constant RBE of 1.1 instead of a variable RBE will lead to an underestimate of expected NTCP values for organs at risk making proton plan appear more favorable compared to photon plans than they really are.

In conclusion, we found that similar treatment plans can be generated with IMPT compared to VMAT in terms of target coverage, target conformity, and OAR sparing, when range and HU uncertainties are neglected. However, when accounting for these uncertainties during robust optimization, VMAT outperforms IMPT in terms of achievable target conformity and OAR sparing.

\section{REFERENCES}

1. NIH National Cancer Institute. Surveillance, epidemiology, and end results program. Cancer stat facts: prostate cancer. 2017. Available from: https://seer.cancer.gov/ statfacts/html/prost.html [cited 02/10/2017].

2. Hegemann NS, Guckenberger M, Belka C, Ganswindt U, Manapov F, Li M. Hypofractionated radiotherapy for prostate cancer. Radiat Oncol 2014; 9: 275. doi: https://doi.org/10.1186/s13014-014-0275-6

3. Brenner DJ, Martinez AA, Edmundson GK, Mitchell C, Thames HD, Armour EP. Direct evidence that prostate tumors show high sensitivity to fractionation (low alpha/beta ratio), similar to late-responding normal tissue. Int J Radiat Oncol Biol Phys 2002; 52: 6-13. doi: https://doi.org/10.1016/S03603016(01)02664-5

4. Fowler JF. The radiobiology of prostate cancer including new aspects of fractionated radiotherapy. Acta Oncol 2005; 44: 265-76. doi: https://doi.org/10.1080/ 02841860410002824

5. Halpern JA, Sedrakyan A, Hsu WC, Mao J, Daskivich TJ, Nguyen PL, et al. Use, complications, and costs of stereotactic body radiotherapy for localized prostate cancer. Cancer 2016; 122: 2496-504. doi: https://doi. org/10.1002/cncr.30101

6. NCCN. Clinical practice guidelines in oncology: prostate cancer version 1. 2017 Available from: https://www.nccn.org/ professionals/physician_gls/default.aspx.

7. US National Library of Medicine. Dose wscalation study using ultrahypofractionated, image-guided, intensitymodulated radiotherapy in prostate cancer. 2009. Available from: https://clinicaltrials. gov/ct2/show/NCT00911118.
8. Boike TP, Lotan Y, Cho LC, Brindle J, DeRose P, Xie XJ, et al. Phase I doseescalation study of stereotactic body radiation therapy for low- and intermediaterisk prostate cancer. J Clin Oncol 2011; 29: 2020-6. doi: https://doi.org/10.1200/JCO. 2010.31.4377

9. Kole TP, Nichols RC, Lei S, Wu B, Huh SN, Morris CG, et al. A dosimetric comparison of ultra-hypofractionated passively scattered proton radiotherapy and stereotactic body radiotherapy (SBRT) in the definitive treatment of localized prostate cancer. Acta Oncol 2015; 54: 825-31. doi: https://doi.org/ 10.3109/0284186X.2014.953260

10. Unkelbach J, Chan TC, Bortfeld T, Jan U, Timothy CYC, Thomas B. Accounting for range uncertainties in the optimization of intensity modulated proton therapy. Phys Med Biol 2007; 52: 2755-73. doi: https://doi. org/10.1088/0031-9155/52/10/009

11. Patel RR, Orton N, Tomé WA, Chappell $\mathrm{R}$, Ritter MA. Rectal dose sparing with a balloon catheter and ultrasound localization in conformal radiation therapy for prostate cancer. Radiother Oncol 2003; 67: 285-94. doi: https://doi.org/10.1016/S01678140(03)00056-2

12. Yang M, Zhu XR, Park PC, Titt U, Mohan R, Virshup G, et al. Comprehensive analysis of proton range uncertainties related to patient stopping-power-ratio estimation using the stoichiometric calibration. Phys Med Biol 2012; 57: 4095-115. doi: https://doi.org/10. 1088/0031-9155/57/13/4095

13. Hansen DC, Seco J, Sørensen TS, Petersen JB, Wildberger JE, Verhaegen F, et al. A simulation study on proton computed tomography (CT) stopping power accuracy using dual energy CT scans as benchmark. Acta Oncol 2015; 54: 1638-42. doi: https:// doi.org/10.3109/0284186X.2015.1061212

14. Wang D, Mackie TR, Tomé WA. On proton CT reconstruction using MVCT-converted virtual proton projections. Med Phys 2012; 39: 6Part1: 2997-3008. doi: https://doi.org/ $10.1118 / 1.4711752$

15. Slater JD, Yonemoto LT, Rossi CJ, Reyes-Molyneux NJ, Bush DA, Antoine JE, et al. Conformal proton therapy for prostate carcinoma. Int J Radiat Oncol Biol Phys 1998; 42: 299-304. doi: https://doi.org/10.1016/ S0360-3016(98)00225-9

16. IRCU. IRCU report 83: prescribing, recording, and reporting photon-beam intensitymodulated radiation therapy (IMRT). vol. 10: Journal of the International Commission on Radiation Units and Measurements; 2010. pp. 1-2.

17. Soisson ET, Mehta MP, Tomé WA. A comparison of helical tomotherapy to circular collimator-based linear-accelerator radiosurgery for the treatment of brain metastases. Am J Clin Oncol 2011; 34: 388-94. doi: https://doi.org/10.1097/COC. 0b013e3181e9c0ee

18. Hardcastle N, Metcalfe PE, Rosenfeld AB, Tomé WA. Endo-rectal balloon cavity dosimetry in a phantom: performance under IMRT and helical tomotherapy beams. Radiother Oncol 2009; 92: 48-56. doi: https:// doi.org/10.1016/j.radonc.2009.03.004

19. Ödén J, Eriksson K, Toma-Dasu I. Inclusion of a variable RBE into proton and photon plan comparison for various fractionation schedules in prostate radiation therapy. Med Phys 2017; 44: 810-22. doi: https://doi.org/ 10.1002/mp.12117 